\title{
Szenariobasierte Analyse der Kurzschlussströme im deutschen Niederspannungsnetz unter Verwendung der CIGRE-Referenznetze
}

\author{
M. Plenz(D, F. Grumm, M. F. Meyer, S. Boden, D. Schulz, K. Lehmann
}

\begin{abstract}
Die fortlaufenden, regulatorischen Eingriffe der Bundesregierung im Bereich erneuerbarer Energien führen zu einem hohen Anteil an Windkraft und Photovoltaikanlagen im elektrischen Energieversorgungssystem. Neben einer bevorzugten Einspeisung der erneuerbaren Energien erfolgt aktuell und zukünftig ein Rückbau oder die Stilllegung konventioneller Kraftwerke, wie Atom- und Kohlekraftwerke. Als quantitativer Bewertungsparameter zur Abschätzung der potenziellen Konsequenzen wird die Kurzschlussimpedanz verwendet, die aus den Kurzschlussströmen ableitbar ist. Die Kurzschlussimpedanz dient beispielsweise zur Bewertung der Aufnahmekapazität der Netzanschlusspunkte, der Sicherstellung der Versorgungsqualität und der Reduktion der Netzrückwirkungen. Die Impedanz eines Netzverknüpfungspunktes einer Anlage berechnet sich aus Netzkurzschlussleistung und der Netznennspannung. Somit ist die Netzkurzschlussleistung ein wesentlicher Parameter der Netzbewertung, der sich durch die Energiewende verändert. Betrachtet wird in diesem Beitrag der Anfangs-Kurzschlusswechselstrom, der die Grundlage für die rechnerische Größe der Netzkurzschlussleistung ist.

Dieser Beitrag analysiert den Einfluss der Veränderung der Kurzschlussströme auf die Niederspannung (NS) unter Verwendung von Referenznetzen der Nieder- und Mittelspannungsebene (MS), szenariobasierten Simulationen und vereinfachten Modellen für erneuerbare Energieanlagen.

Gleichzeitig werden bisherige Forschungsergebnisse auf Tendenzen untersucht, die die Veränderungen der Kurzschlussströme und den Einfluss von EE-Anlagen analysieren. Die Ergebnisse der Simulationen zeigen, dass die Kurzschlussströme trotz der Abschaltung von Großkraftwerken, die bisher durch Synchrongeneratoren den größten Anteil am Netzkurzschlussstrom lieferten, nicht in relevantem Maße abnehmen.
\end{abstract}

Schlüsselwörter: Kurzschlussstrom; Netzstabilität; Simulation; Prognose; Netzstörung; erneuerbare Energien

Influence of RE on short-circuit currents in the low-voltage distribution network.

The massive German government support for renewable energies is leading to changes in different areas, e.g., energy system management. The high share of wind power and photovoltaic systems is feeding much energy into the electrical energy supply grid. In addition to preferential treatment for the energy supply of the renewable energy plants, a throttling performance or the shutdown of conventional power plants i.e., nuclear or coal power plants takes place. This could lead to negative effects on the short-circuit capacity. The short-circuit capacity is necessary, for example, to quantify the hosting capacity of renewable generation or the impact on power quality. The paper analyzes the influence with a combination of scenario-based simulations and reference grids. The paper approach shows that the low-voltage short-circuit currents will not reduce in a relevant amount due to the shutdown of large power plants.

Keywords: short-circuit current; grid stability; grid simulation; grid perturbations; renewable energies

Eingegangen am 13. Dezember 2019, angenommen am 14. April 2021, online publiziert am 22. April 2021

() The Author(s) 2021

\section{Einleitung}

Im Bereich der Energieversorgung steht die Gesellschaft weltweit vor großen Herausforderungen. National bedarf es von jedem Land Anstrengungen, dem Klimawandel entgegenzutreten. In Deutschland führt die staatliche Förderung der erneuerbaren Energien (EE) zu einem hohen Anteil an Windkraftanlagen (WKA) und Photovoltaikanlagen (PVA) im elektrischen Energieversorgungsnetz. Aufgrund der Vorzugsbehandlung bei der Energieeinspeisung der EE-Anlagen erfolgt eine Leistungsdrosselung konventioneller Kraftwerke. Bis zum Jahr 2050 strebt die deutsche Bundesregierung einen Stromversorgungsanteil von $80 \%$ aus erneuerbaren Energiequellen an [1].
Die Autoren M. Plenz and F. Grumm haben in gleichem Maße an dem Beitrag mitgewirkt.

Plenz, Maik, Fachgebiet Elektrische Energiesysteme, Helmut-Schmidt-Universität Hamburg, Hamburg, Germany (E-Mail: maik.plenz@hsu-hh.de); Grumm, Florian, Fachgebiet Elektrische Energiesysteme, Helmut-Schmidt-Universität Hamburg, Hamburg, Germany; Meyer, Marc Florian, Fachgebiet Elektrische Energiesysteme, Helmut-Schmidt-Universität Hamburg, Hamburg, Germany; Boden, Scott, Fachgebiet Elektrische Energietechnik, Brandenburgische Technische Universität Cottbus-Senftenberg, Senftenberg, Germany; Schulz, Detlef, Fachgebiet Elektrische Energiesysteme, Helmut-Schmidt-Universität Hamburg, Hamburg, Germany; Lehmann, Kathrin, Fachgebiet Elektrische Energietechnik, Brandenburgische Technische Universität Cottbus-Senftenberg, Senftenberg, Germany 
Um eine Low-Carbon-Economy zu etablieren, sollen bis 2022 nur noch 15 GVA installierte Leistung aus Kohlekraftwerken am Netz sein, bis spätestens 2038 sollen alle Kohlekraftwerke abgeschaltet werden. Zusätzlich steht Deutschland mit dem selbstgewählten Ansatz des Austritts aus der Kernenergie vor der sukzessiven Abschaltung von Atomkraftwerken mit einer installierten Gesamtleistung von ca. 10 GVA [2].

Die Entwicklung der Kurzschlussströme im Zuge des Zubaus der regenerativen Energien wird in diesem Artikel in den Fokus gestellt. Aus dem Anfangs-Kurzschlusswechselstrom ist die Netzkurzschlussleistung errechenbar.

$$
S_{k}^{\prime \prime}=\sqrt{3} \cdot U_{n} \cdot l_{k}^{\prime \prime}
$$

Die Netzkurzschlussleistung $S_{k}^{\prime \prime}$ ist eine reine Rechengröße, die in vielen Normen als Grundlage für die Berechnung der Impedanz eines Netzanschluss-bzw. Netzverknüpfungspunktes (NVP) dient, vergl. z. B. VDE-AR-4105, VDE-AR-4110. Für die Berechnung von Kurzschlussströmen wird sie nicht mehr angewandt, vergleiche DIN EN 60909-0, Kapitel 6.2. Dennoch geben viele Netzbetreiber anstelle der Anfangs-Kurzschlusswechselströme die Kurzschlussleistung an. Daher und aufgrund der Normen wird sie in diesem Artikel als Bezugsgröße verwandt [17].

$$
Z_{\mathrm{NVP}}=\frac{c \cdot U^{2}}{S_{k}^{\prime \prime}}
$$

Diese Netzimpedanz eines NVP dient der Quantifizierung von Netzrückwirkungen wie Harmonischen und Flicker.

Der Kurzschlussstrom hingegen bestimmt u.a. das Schaltvermögen von Leistungsschaltern und die Beanspruchung von elektrischen Anlagen. Somit ist der Kurzschlussstrom eine relevante Größe zur Bewertung eines NVP oder eines Netzabschnittes.

Die großen Synchronmaschinen der konventionellen Kraftwerke besitzen durch das Prinzip der Erhaltung des magnetischen Flusses bei rotierenden elektrischen Maschinen im Fehlerfall bzw. den Reaktanzen der Maschine bei transienten Vorgängen einen weit höheren Kurzschlussstrom als WKA oder PVA bezogen auf den Bemessungsstrom der elektrischen Erzeugungsanlage. Dies spiegelt sich wieder in den Normen und Anwendungsregeln: Die Anschlussregel für die Niederspannung VDE-AR-N-4105 gibt bei WKA und PVA, die leistungselektronisch gekoppelt sind, gilt das Einfache des Bemessungsstromes [3]. Für Windparks ergeben sich höhere Ströme, je nach dem technischen Konzept der Erzeugungseinheiten.

Die Auswirkung, dieser von staatlicher Förderung und Umweltschutzmaßnahmen getriebenen Entwicklung bei gleichzeitiger Stilllegung konventioneller Anlagen, auf die Kurzschlussströme auf unterlagerten Netzebenen und hier auf der Niederspannungsebene wird mit Szenarien analysiert. Dafür werden relevante Einflussfaktoren und deren Ergebnisse zusammengefasst. Szenario-basierte Kurzschluss-Analysen sollen die Hypothese belegen, dass sich die Höhe der Kurzschlussströme im Verteilnetz durch die zunehmende Integration von EE-Anlagen in der Energiewende nicht bzw. positiv verändert. Dabei werden bisherige Ergebnisse auf Tendenzen untersucht, die Veränderungen der Kurzschlussströme und der Einfluss der EE-Anlagen auf Referenznetze analysiert. In diesem Artikel wird angenommen, dass ausschließlich Erzeugungseinheiten mit Vollumrichtern eingesetzt werden. Dies ist die untere Schranke für die Entwicklung der maximalen Kurzschlussströme, denn Vollumrichter liefern den geringsten Kurzschlussstrom aller Anlagen-Typen von EEAnlagen.

\subsection{Vergleich des Kurzschlussverhaltens konventioneller rotierender Generatoren und Erzeugungseinheiten mit Vollumrichtern}

Das Kurzschlussverhalten von Synchronmaschinen unterscheidet sich physikalisch von leistungselektronisch gekoppelten Anlagen mit Wechselrichtern bzw. Vollumrichtern. Letztere sind durch eine elektronische Regelung und elektronische Schutzfunktionen bestimmt, die einen unzulässig hohen Energieeintrag in den Zwischenkreiskondensator und das schaltende Leistungshalbleitermodul sowie dessen Zerstörung verhindern. Unterschieden wird in langsame und schnelle Schutzverfahren: Langsame Schutzverfahren basieren auf der Regelung und Schnellschutzverfahren sind direkt in die Treiber der Leistungshalbleitermodule integriert. Die Schnellschutzverfahren begrenzen Kurzschlussströme durch die Halbleitermodule innerhalb von einigen Mikrosekunden.

Messungen des maximalen Kurzschlussstroms zeigen, dass dieser zwischen dem einfachen bis 1,5-Fachen des Bemessungsstromes liegt [4]; dies ist abhängig von der Regelungsgeschwindigkeit und der Stromtragfähigkeit der Leistungshalbleiter.

Dieses zeigt auch die Kurzschluss-Messung eines Niederspannungswechselrichters aus Abb. 1. Gemessen wird ein dreipoliger Klemmenkurzschluss eines dreiphasigen transformatorlosen Wechselrichters unter ohmsch-geprägten Bedingungen. Die Blindleistungseinspeisung ist vernachlässigbar, da die Zeitkonstante der Q(U)-Regelung zwischen 10 s und 60 s beträgt [3].

Bei Eintreten des Kurzschlusses entlädt sich der Kondensator des Netzfilters: Es tritt eine transienter Entladestrompuls auf. Die Pulsenergie ist nur gering, daher kann diese Transiente vernachlässigt werden [4]. Nach Fehlereintritt speist der Wechselrichter weiterhin einen nahezu sinusförmigen Strom ein.

Aufgrund der Abregelung des Wechselrichters ergibt sich das vereinfachte Ersatzschaltbild nach der EN 60909-0 [14] aus Abb. 2 für einen dreipoligen Fehler: eine Stromquelle im Mitsystem mit meist unendlichen Impedanzen im Null- und Gegensystem.

Bei Synchronmaschinen bestimmen die transiente und die subtransiente Übergangsreaktanz den Ausgleichsvorgang bei einem Klemmenkurzschluss und damit den Kurzschlussstrom. Die treibende Spannung ist von der Vorbelastung des Generators abhängig. Es ergibt sich das Ersatzschaltbild aus Abb. 2. Eine Übersicht zum Verhalten der gängigen Generator-Konzepte im Fehlerfall findet sich in [15]. Da die Abbildung mit einer Stromquelle in Simulationsumgebungen problematisch sein kann, wird ein generisches Modell genutzt. Dies beruht auf dem Thévenin-Äquivalent mit einer Spannungsquelle und einer Reaktanz, die so angepasst sind, dass der maximale Fehlerstrom (Nennstrom der Anlage) fließt.

\section{Einflussfaktoren auf den Kurzschlussstrom der Mittel- und Niederspannungsverteilnetze und Prognosen zur Entwicklung}

Folgende Faktoren beeinflussen unter anderem den Kurzschlussstrom in der NS: die Kurzschlussleistung des Übertragungsnetzes, Netzimpedanzen im NS-Netz, EE-Anlagen und eine ungewollte Inselnetzbildung [5]. Diese Faktoren gelten ebenso für die Mittelspannungsebene.

\subsection{Entwicklung der Kurschlussströme im deutschen Übertragungsnetz}

Die stetige Reduktion der Einspeiseleistung konventioneller Kraftwerke bei gleichzeitiger Erhöhung volatiler Erzeugungsanlagen mit leistungselektronischen Netzkopplungen mit geringeren Kurzschlussströmen, führt zu den Annahmen, dass: 


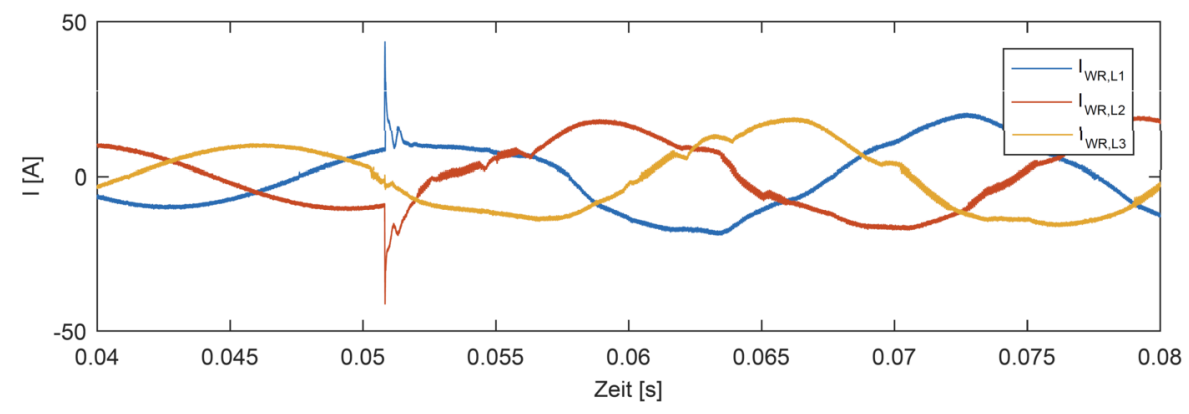

Abb. 1. Messung eines dreipoligen Klemmenkurzschlusses eines dreiphasigen transformatorlosen Wechselrichters in einer ohmsch-geprägten Testumgebung Sunny Boy 5000 (Bemessungsleistung 5 kW)

Wechselrichter / Vollumrichter

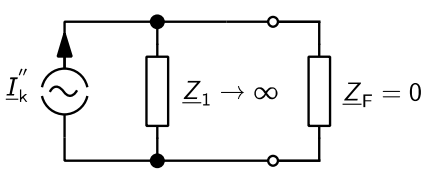

Synchrongenerator

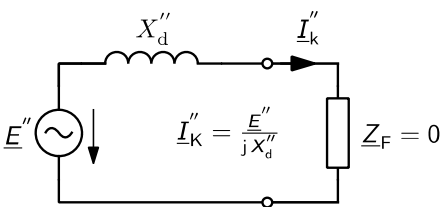

generisches Ersatzschaltbild

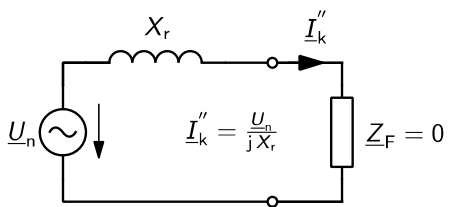

Abb. 2. Ersatzschaltbilder zur Berechnung der Kurzschlussströme bei Klemmenkurzschluss

- die Kurzschlussleistung $\left(S_{k}^{\prime \prime}\right)$ des elektrischen Netzes sinkt und damit nimmt die Kurschlussimpedanz zu;

- sich somit ebenfalls der Anfangs-Kurzschlusswechselstrom $\left(l_{k}^{\prime \prime}\right)$ verringert und

- der Kurzschlussstrom starken Schwankungen in Abhängigkeit von Tageszeit und Wetter unterliegt, da die Einspeisung von großen Wind- und PV-Parks Verhältnis zu den konventionellen Kraftwerken zunimmt.

Es ist zu untersuchen, ob die Annahmen zutreffend sind oder ob die verbleibenden konventionellen Kraftwerke weiter ausreichend hohe Kurzschlussströme liefern können [6, 7], so dass die Impedanz des NVP im Fehlerfall nicht zu hoch wird, siehe Formel $Z_{\text {NVP. }}$. Eine Meta-Analyse liefert eine Richtung zur Entwicklung der Kurschlussströme. Eine Zusammenfassung der Ergebnisse erfolgt in Tab. 1. In den folgenden Ausführungen bezeichnet die Kurzschlussimpedanz die 50-Hz-Impedanz des Netzes bzw. des Netzanschlusspunktes im Kurzschlussfall.

Die Ergebnisse aus [8] zeigen, dass bei der Kurschlussimpedanz keine tendenziellen Änderungen auftreten und die Stabilität nicht gefährdet wird. Sowohl die im Netzgebiet von [8] untersuchten heutigen Knoten mit minimaler und maximaler Kurzschlussimpedanz werden im Zukunftsszenario 2033 weder unter- noch überschritten. Es wird keine zusätzliche Kurzschlussleistung durch das Ausland bereitgestellt und der minimale Anfangs-Kurzschlusswechselstrom der meisten Knoten nimmt zu. Wie zu erwarten, ist der AnfangsKurzschlusswechselstrom stark wetterabhängig und temporär volatil. Der Spannungstrichter um den Fehlerort weitet sich im Vergleich zum bestehenden System aus [6-9]. Jedoch tragen aufgrund des ausgeweiteten Spannungstrichters mehr EE-Anlagen zum Kurzschlussstrom bei.

EE-Anlagen in der NS besitzen ebenfalls ein Fault-Ride-Through (FRT) Verhalten. Diese FRT-Anforderungen definieren die Anlagenstromeinspeisung bzw. das Durchfahren von Fehlerzuständen auf Grundlage der am Netzanschlusspunkt (NAP) vorherrschenden Spannung in Abhängigkeit der Zeit. Die Anforderungen in der Nie- derspannung unterscheiden sich von den Anforderungen für Anlagen in der MS-Ebene. Bei einer Spannung kleiner 0, $8 U_{n}$ fordert die VDE AR4105 zur Vermeidung von "Blinding of Protection" also die Auslöseverzögerung von Sicherungselementen in der Niederspannung, dass Wechselrichteranlagen am Netz verbleiben, jedoch den eingespeisten Strom auf null reduzieren [3]. Diese Bedingung ist bei einem Kurzschluss erfüllt.

Die Studie [10] zeigt, dass sich die Kurschlussimpedanz in den Übertragungsnetzen nur geringfügig reduziert. Als Gründe werden der Verbleib einiger Synchrongeneratoren im Netz und der Kurzschlussbeitrag aus dem Ausland aufgeführt. Der Kurzschlussbeitrag aus dem Ausland ist von großer Relevanz und eine Umstrukturierung der Stromnetze von Ländern, die mit dem deutschen Stromnetz gekoppelt sind, führt zu veränderten Anforderungen an die Mindesterzeugung der EE-Anlagen [10].

Die Studie [11] beschäftigte sich bei der Veränderung der Kurschlussimpedanz mit den Folgen der Abschaltung konventioneller Kraftwerke. In einem generischen Verteilnetz weisen die Ergebnisse auf eine Senkung um bis zu $14 \%$ hin. Die Abschaltung der Kernkraftwerke kann diese Senkung beschleunigen. In der Studie wird außerdem prognostiziert, dass das vorherige dynamische Netzstabilitätsniveau aufgrund Ersetzung konventioneller Kraftwerke durch EE-Anlagen gesichert werden kann [11].

Die Studien treffen unterschiedliche Aussagen zum Kurzschlussstrom: Zum einem der Kurzschlussstrom wird zu nehmen und zum anderen der Kurzschlussstrom wird sinken. Dies liegt an den unterschiedlichen Faktoren, die sie bei der Beurteilung der Veränderung der Kurschlussströme berücksichtigen. Es werden z. B. unterschiedliche Zeiträume, variierende Annahmen für den Netzausbau und verschiedene Untersuchungsmethoden verwendet. Die grundlegende Gemeinsamkeit liegt in der Veränderung der Kurschlussströme aufgrund der Abnahme konventioneller Kraftwerke. Daher erfolgt eine erneute Betrachtung dieses Themas mit Referenznetzen. 
Tab. 1. Vergleich der Studienanalysen

\begin{tabular}{lll}
\hline Studie [Quelle] & Annahmen & Tendenz \\
\hline Deutsche Energie-Agentur & Netz: & Durchschnittlicher Anstieg der \\
GmbH (dena), "dena-Studie & - Übertragungsnetzmodell kombiniert mit & maximalen Kurzschlussleistung um \\
Systemdienstleistung 2030" [8] & beispielhaften 110 kV Verteilnetzmodell & $25 \%$ im ländlich geprägten \\
& Ziel: & Verteilnetz \\
& - Betrachtung von maximaler und minimaler & Starke Variation der \\
& konventioneller Erzeugung in Relation zu den & Kurzschlussleistung im industriell \\
& Witterungsbedingungen & geprägten Verteilnetz (Anstiege bis \\
& - Betrachtung eines ländlichen und industriell & zu +80 \% und Senkungen bis zu \\
& geprägten Verteilnetzes & $-40 \%$ je nach Knotenpunkt)
\end{tabular}

FGH: „Studie zur Ermittlung der technischen Mindesterzeugung des konventionellen Kraftwerksparks zur Gewährleistung der Systemstabilität in den deutschen Übertragungsnetzen bei hoher Einspeisung aus erneuerbaren Energien" [10]

Universität Kassel Studie: "Studie zur Veränderung der Kurzschlussleistung beim Übergang von einem zentral zu einem dezentral gespeisten Energieversorgungssystem: Endbericht zum Verbundvorhaben" [11]
-2011 bis 2033

Netz:

- Betrachtung des konventionellen deutschen Kraftwerkparks im Regionenmodell 2013 (Übertragungsnetz)

Ziel:

- Methoden zur Bestimmung der

Mindestbereitstellung von Kurzschlussleistung

- Verbleib von einigen großen

Synchrongeneratoren im Netz und Einfluss der Kurzschlussleistung des Auslands

Zeitraum:

Nicht definiert

Netz:

- Energieversorgungsgebiet Kassel und

Umgebung bestehend aus synthetischen 380/110 kV Netzen und realen MS- und NSNetzen

Ziel:

- Berechnung der Kurzschlussleistung auf Basis von Prognosen der Veränderung der Generatortypen im Stromnetz

Zeitraum:

2010 bis 2032
2.2 Analytische Betrachtung des Einfluss des Übertragungsnetzes auf die Kurschlussimpedanz im deutschen Verteilnetzen der Mittel- und Niederspannungsebene

Wie erwähnt, benennt die Studie des Fraunhofer IWES [11] einen Verlust von $20 \%$ der Kurschlussleistung des Niederspannungsnetzes im Jahre 2032 bezogen auf die Istwerte des Jahres 2011, ohne die Netzverstärkungsmaßnahmen aufgrund der EE-Anlagen miteinzubeziehen. Damit würde die Kurzschlussimpedanz der NVP steigen und die Kurzschlussströme sinken. Diese Verstärkungsmaßnahmen (speziell bei Transformatoren) führen zu einer verbesserten Netzkopplung der Spannungsebenen und folglich zu einer Verringerung der Kurschlussimpedanz, wie es eine Studie von TenneT TSO feststellte [10]

Abbildung 3 zeigt das Netzebenen-Modell für ein einfaches Gedankenexperiment aus [9] zur Abschätzung der Veränderungen auf die Niederspannungsverteilnetze, die typischerweise über drei Transformatoren mit dem Übertragungsnetz gekoppelt sind. Die obere Grenze des Kurzschlussstromes, der sich aus der Kurschlussimpedanz des Übertragungsnetzes speist, ermittelt sich aus einem stark vereinfachten Ersatzschaltbild, das die Abb. 4 zeigt. Die Werte für die Kurzschlussleistung des Übertragungsnetzes zwischen 7 GVA und 28 GVA sind Jahresmittel aus der Studie [16], die Werte wurden ohne EE-Anlagen berechnet. Sie stellen somit Minimalwerte da.

Aus der Betrachtung der Abhängigkeit von $I_{k}^{\prime \prime}$ auf der Unterspannungsseite des Transformators T3 aus Abb. 3 von der Kurzschlussleistung des Übertragungsnetzes $S_{k}^{\prime \prime}$ lässt sich ableiten, dass der Einfluss des Übertragungsnetzes sehr gering ist (Abb. 5). Weiterhin lässt sich Folgendes festhalten:

- Der Einfluss der Kurschlussleistung auf den Anfangs-Kurzschlusswechselstrom verringert sich stark mit größerem Abstand des Fehlers von der Ortsnetzstation, da die Kurzschlussimpedanz von der Niederspannungsleitung bestimmt wird.

- Der Einfluss der Kurschlussleistung auf den Anfangs-Kurzschlusswechselstrom nimmt bei einem Einsatz von Transformatoren mit kleinerer Nennscheinleistung weiter ab, da die Kurzschlussimpedanz steigt.

Das bedeutet, dass die Längsimpedanzen der Transformatoren und Kabel die Impedanz der Fehlerschleife aus Abb. 4 bestimmen, da sie ein Vielfaches der Netzimpedanz betragen [4, 9]. Diese Impedanzen nehmen zukünftig ab, da: 


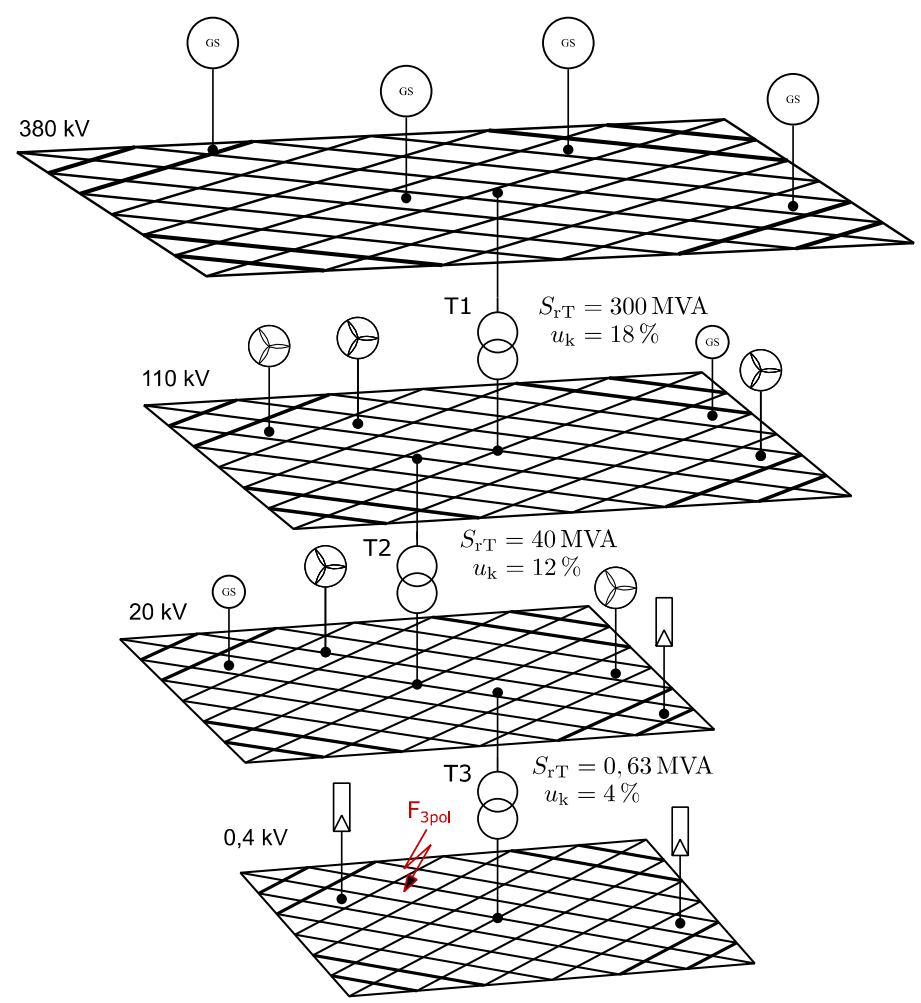

Abb. 3. Vereinfachte Darstellung der Netzverknüpfungen

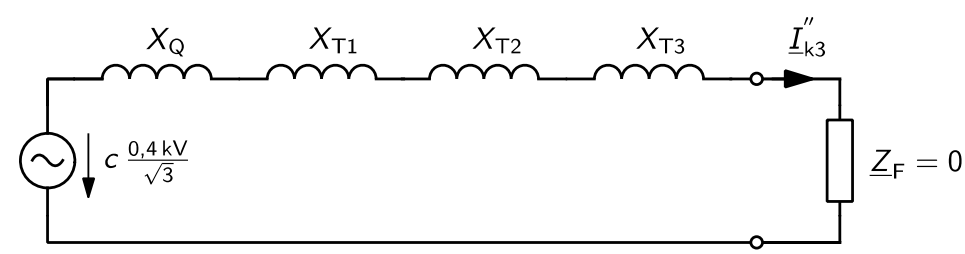

Abb. 4. Ersatzschaltbild der Mitimpedanzen aus dem Gedankenexperiments. $X_{Q}$ : Von der Kurzschlussleistung abhängige Reaktanz des Übertragungsnetzes; $X_{T 1}$ : Reaktanz des Netzkuppelransformators $400 \mathrm{kV} / 110 \mathrm{kV} ; X_{T 2}$ : Reaktanz des Verteilnetztransformators $110 \mathrm{kV} / 20 \mathrm{kV} ; X_{T 3}$ : Reaktanz des Ortsnetztransformators $20 \mathrm{kV} / 0,4 \mathrm{kV} ; c=1$, 1: Spannungsüberhöhungsfaktor für NS

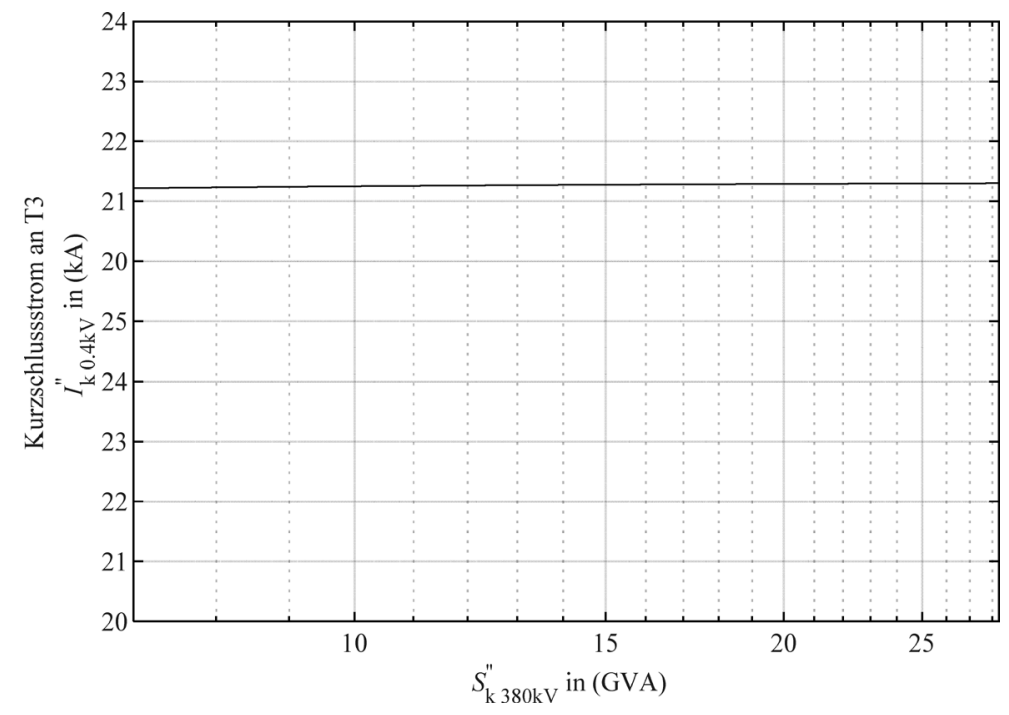

Abb. 5. Kurzschlussstrom auf der Unterspannungsseite des Ortsnetztransformators der Niederspannung in Abhängigkeit von der Kurschlussimpedanz des Übertragungsnetzes 
Tab. 2. Szenarien der Simulation

\begin{tabular}{ll}
\hline Szenario & $\begin{array}{l}\text { Anteil von regenerativen Quellen } \\
\text { an der Gesamtheit der NAP der } \\
\text { betrachteten CIGRE Netze }\end{array}$ \\
\hline 1 & $0 \%$ \\
2 & $30 \%$ \\
3 & $50 \%$ \\
4 & $80 \%$ \\
\hline
\end{tabular}

- Netzverstärkungsmaßnahmen bedingt durch den EE-Ausbau vorgenommen werden.

- Transformatoren in Umspannwerken ersetzt werden und der Ersatz eine höhere Bemessungsscheinleistung vorweist.

- Die Öko-Design Richtlinie 2009/125/EG des Europäischen Parlaments eine Verbesserung der Kurzschlussverluste bei Transformatoren vorschreibt. Dies wirkt sich aber nur geringfügig auf die Kurzschlussspannung aus, da der Anteil der der ohmschen Verluste am $u_{k}$ gering ist.

\section{Einfluss des EE-Ausbaus auf die Entwicklung der Kurzschlussleistung im Verteilnetz}

Der resultierende EE-Einfluss auf die Kurschlussströme wird in einer Simulation untersucht. WKA mit doppelt gespeisten Asynchronmaschinen (DGASM) werden in dieser Analyse nicht betrachtet, da wie in Absch. 1.1 erwähnt ein Worst-Case-Szenario untersucht wird bzw. die untere Schranke für den Anstieg der Kurzschlussströme gesucht wird. Somit ergibt sich mit der ausschließlichen Betrachtung von Wechselrichter bzw. Vollumrichter gekoppelten Anlagen die untere Grenze für die Veränderung des AnfangsKurzschlusswechselstrom.

\subsection{Analysemethodik der Szenarien-basierten Evaluierung} Für die Analyse werden grundlegende Annahmen getroffen:

(1.) Es werden nur Vollumrichteranlagen betrachtet, da diese den geringsten Kurzschlussbeitrag liefern.

(2.) Das Mittelspannungsnetz wird als offenes Ringnetz betrieben, da dass der in der Praxis übliche Fall ist [12].

(3.) Es wird davon ausgegangen, dass die PVA auf der NS einspeisen, da die Messungen gezeigt haben, dass die Abschaltung erst nach 40 ms erfolgt [4]. Somit ist der Kurzschlussstrom für zwei Perioden erhöht. Dies wirkt sich auf den AnfangsKurzschlusswechselstrom aus. In der Simulation wurde die PV Anlage nach dem generischen Ersatzschaltbild berechnet und solange die Kurzschlussreaktanz angepasst bis der Nennstrom der Anlage fließt.

Zur genaueren Analyse der Auswirkungen wird auf Referenznetze zurückgegriffen und für die EE-Anlagen auf das vereinfachte Modell aus Abschn. 1.1. Es werden ein Mittelspannungs- und ein Niederspannungsnetz nach der Vorlage des Cigre Task Force [13] in DlgSILENT PowerFactory konstruiert und mit vier Szenarien berechnet (Tab. 2).

Die Anschlusspunkte der für die jeweiligen Szenarien zugebauten WKA PVA sind entsprechend des maximalen Einflusses auf die Anfangs-Kurzschlusswechselströme nach Kapitel 3.3. bestimmt. Dort wird ein kombiniertes Netz simuliert, um den größten Einfluss auf die Größe der Kurzschlussströme zu berechnen. In dem Netz wird der dreipolige Kurzschluss aller Sammelschienen für alle Szenarien entsprechend der IEC 60909-0 berechnet (siehe Formel 1 2), mit dem Unterschied, dass die Anlagen als Spannungsquelle mit angepasster Reaktanz angenommen werden, siehe Abb. 4 (generisches Ersatzschaltbild), wobei die Mit- gleich der Gegenimpedanz ist und die Nullimpedanz unendlich ist. Unter Kurzschluss wird dabei eine leitfähige Verbindung zwischen zwei Punkten unterschiedlichen Potentials verstanden [14]. Die Schaltzustände wurden nicht verändert, da exakte Berechnungen nur an konkreten Netzen durchführbar sind. Die Netznennspannung $U_{\mathrm{nN}}$ wird noch mit dem minimalen oder maximalen Spannungsüberhöhungsfaktor c aus [14] multipliziert - für NS-Netze gilt $c_{\min }=0,95$ und $c_{\max }=1,1$ und für MS-Netze $c_{\min }=1$ und $c_{\max }=1,1$. Beim dreipoligen Kurzschluss ist nur die Mitimpedanz von Interesse. Die resultierenden Werte der Kurzschlussströme werden auf die Referenzwerte des Szenarios 1 bezogen.

$$
\begin{aligned}
& l_{\mathrm{k} 3 \min }^{\prime \prime}=\frac{C_{\min } \cdot U_{\mathrm{nN}}}{\sqrt{3} \cdot Z_{\mathrm{k}(1) \max }} \\
& I_{\mathrm{k} 3 \max }^{\prime \prime}=\frac{C_{\max } \cdot U_{\mathrm{nN}}}{\sqrt{3} \cdot Z_{\mathrm{k}(1) \min }}
\end{aligned}
$$

\subsection{Die CIGRE-Referenznetze}

Die für die Simulation verwendeten Netze werden mit dem Benchmark System der "CIGRE Task Force" erstellt [13]. Dieses BenchmarkSystem ist eine Vereinfachung für Analysen zu Auswirkungen der zunehmenden Integration von EE in das elektrische Energieversorgungsystem. Das Benchmark-System wird in Form von allgemeinen Hoch-, Mittel- und Niederspannungsnetzes nach europäischen und nordamerikanischen Standards erstellt. Für die Simulation werden die europäischen Referenznetze der Mittel - und des Niederspannungsnetze verwendet (siehe Abb. 7 und Abb. 8). Alle Betriebsmittelparameter können in [13] eingesehen werden. In dem Niederspannungsreferenznetz werden nur Industrie- und Haushaltskunden analysiert.

\subsection{Methodik zur Bestimmung der Netzanschlusspunkte der WKA / PVA mit dem größten Einfluss auf den Kurzschlussstrom}

Es wird die Veränderung der Kurschlussstrom aller Sammelschienen des Mittelspannungsreferenznetzes bei einem dreipoligen Kurzschluss aller Sammelschienen untersucht. Die Sammelschiene, an welcher die größte Veränderung des Kurschlussstromes zwischen den Szenarien auftritt, wird als externes, vorgelagertes Netz des Niederspannungsreferenznetzes verwendet. Das MS-Netz ist vom NSNetz entkoppelt, betrachtet wird nur die mögliche Veränderung der vorgelagerten Kurzschlussleistung des MS-Netzes.

Für die Integration von EE-Anlagen in die simulierten Netze müssen die Anschlusspunkte der WKA und PVA in der MS/NS nach der Formel (3) bestimmt werden. Die verwendeten Parameter listet Tab. 3 auf.

$$
f\left(U_{\mathrm{a}}(k), I_{\mathrm{Aa}}(k), S_{\mathrm{k}}^{\prime \prime}(k)\right)=\min \left\{\frac{U_{\mathrm{a}}(k)}{U_{\mathrm{nN}}}+\frac{I_{\mathrm{Aa}}(k)}{I_{\mathrm{rB}}(k)}+\frac{S_{k}^{\prime \prime}(k)}{S_{\mathrm{kE}}}+\frac{\overline{I_{\mathrm{rB}}}(k)}{I_{\mathrm{rB}}}\right\}
$$

Die Formel (3) bestimmt den optimalen Anschlusspunkt (nach maximalen Einfluss des Anfangs-Kurzschlusswechselstroms) der WKA und PVA. Für die Bestimmung der normierten Formelparameter ist jeweils vor dem Zubau einer neuen Erzeugungseinheit eine Lastflusssimulation sowie eine Kontrolle der Einhaltung der Betriebsbedingungen wie Spannungsgrenzen, Auslastung durchzuführen, zur Bestimmung der in Tab. 3 aufgeführten Faktoren 1, 2 und 4. Zur Berechnung des Faktors 3 wird eine Kurzschlussberechnung eines dreipoligen Kurzschlusses an allen Sammelschienen entsprechend [14] durchgeführt. Es ergibt sich der Ablaufplan aus Abb. 6 zur Ermittlung des optimalen Netzverknüpfungspunktes der nächsten Anlage. 


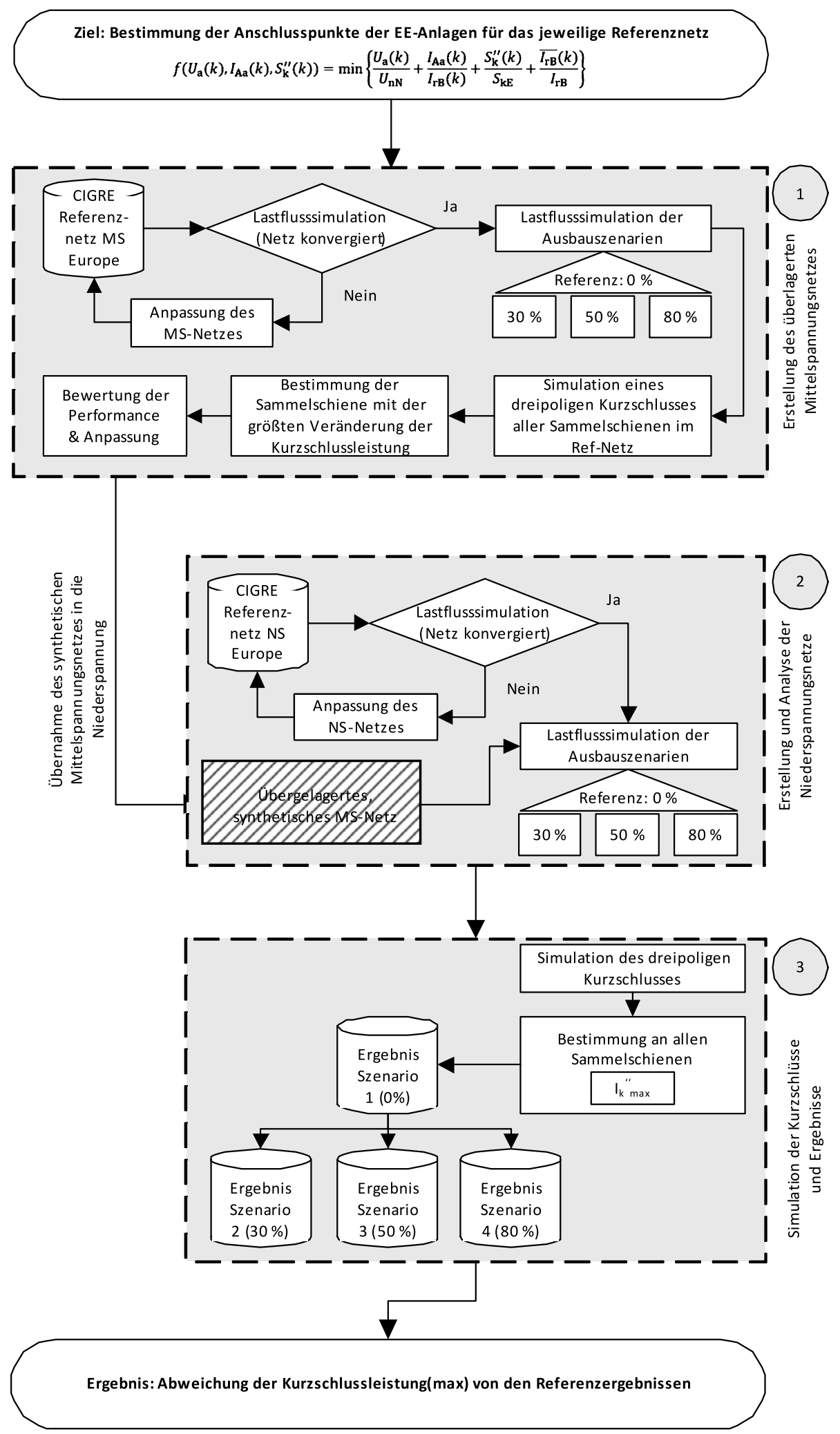

Abb. 6. Ablaufdiagramm zur Bestimmung der kritischsten Netzanschlusspunkte 


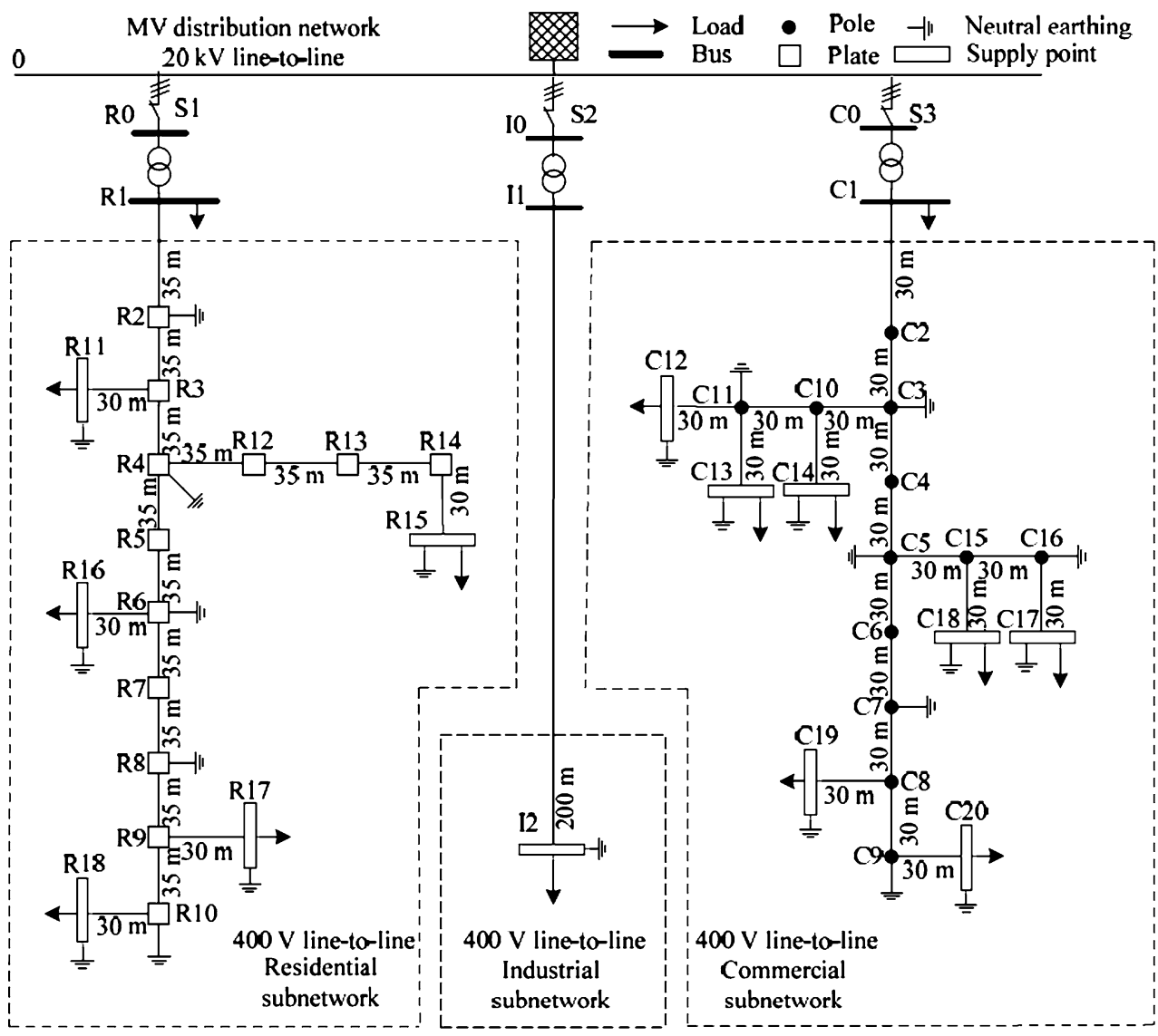

Abb. 7. Europäisches Niederspannungsreferenznetz nach CIGRE [13]

Tab. 3. Normierte Gleichungsparameter für die Bestimmung der Anschlusspunkte

\begin{tabular}{|c|c|c|}
\hline Faktor & Term & Erläuterung \\
\hline 1 & $\frac{U_{a}(k)}{U_{n N}}$ & $\begin{array}{l}\text { Abweichung der Spannung an der } \\
\text { Sammelschiene in p.u. } U_{\mathrm{a}}(\mathrm{k}) \text { ist die } \\
\text { Spannung am Anschlusspunkt } k \text { im } \\
\text { stationären Zustand. } U_{\mathrm{nN}} \text { ist die } \\
\text { Netznennspannung. }\end{array}$ \\
\hline 2 & $\frac{l_{A a}(k)}{l_{r B}}$ & $\begin{array}{l}\text { Bestimmung der } \\
\text { Betriebsmittelauslastung. IAa ist der } \\
\text { Betriebsmittelstrom am Anschlusspunkt } k \\
\text { im stationären Zustand. IrB ist der } \\
\text { Bemessungsstrom des Betriebsmittels }\end{array}$ \\
\hline 3 & $\frac{S_{k}^{\prime \prime}(k)}{S_{k E}}$ & $\begin{array}{l}\text { Bezug der Kurschlussimpedanz } S_{k}^{\prime \prime}(k) \text { des } \\
\text { Anschlusspunktes } k \text { auf die } \\
\text { Kurschlussimpedanz des externe Netzes } \\
S_{\text {kE. }}\end{array}$ \\
\hline 4 & $\frac{\overline{I_{\mathrm{rB}}(k)}}{I_{\mathrm{rB}}}$ & $\begin{array}{l}\text { Bezug des mittleren Nennstroms aller } \\
\text { Kabel } \overline{I_{\mathrm{rB}}}(k) \text { am Anschlusspunkt } k \text { auf den } \\
\text { Nennstrom aller Kabel } I_{\mathrm{rB}} \text { an der } \\
\text { Sammelschiene. }\end{array}$ \\
\hline
\end{tabular}

Schritt 1: Bestimmung der Faktoren 1, 2 und 4 mit einer Lastflusssimulation.
Schritt 2: Bestimmung des Faktors 3 durch Berechnung des dreipoligen Anfangs-Kurzschlusswechselstromes an allen Sammelschienen entsprechend [14].

Schritt 3: Bestimmung des optimalen Anschlusspunktes nach (5).

Schritt 4: Anschluss der neuen Erzeugungseinheit.

Schritt 5: Für die Bestimmung weiterer Anschlusspunkte sind die Schritte 1 bis 4 zu wiederholen.

\subsection{Ergebnisse der Fallstudie}

Die Abb. 9 bis Abb. 11 stellen einen Überblick der Veränderungen der Anfangs-Kurzschlusswechselströme in den Szenarien 2 bis 4, bezogen auf die Werte des Szenarios 1, dar.

In der Abb. 9 ist die Veränderung der maximalen AnfangsKurzschlusswechselströme im Szenario 2 dargestellt, bezogen auf die Werte von Szenario 1. Der Anstieg ist gering: Die maximale Zunahme beträgt 3,6 \% an der Sammelschiene R8. Am kleinsten ist die Zunahme mit 0,012 \% an der Sammelschiene 12. Im Mittel steigt der maximale Anfangs-Kurzschlusswechselstrom der Sammelschienen im Szenario 2 um 2,3 \%.

In der Abb. 10 ist die Veränderung der maximalen AnfangsKurzschlusswechselströme im Szenario 3 dargestellt, bezogen auf die Werte des Szenarios 1. An den Sammelschienen R1 bis R18 ist eine Zunahme der Anfangs-Kurzschlusswechselströme erkennbar. Maximal ist die Zunahme mit 11,07 \% an der Sammelschiene R7. Im Mittel beträgt die Zunahme an den Sammelschienen 11 bis R18 6,3 \% im Szenario 3. Die Zunahme der maximalen AnfangsKurzschlusswechselströme $I_{k \text { max }}^{\prime \prime}$ aus den Ergebnissen der Simulation ist in der Abb. 11 dargestellt. 


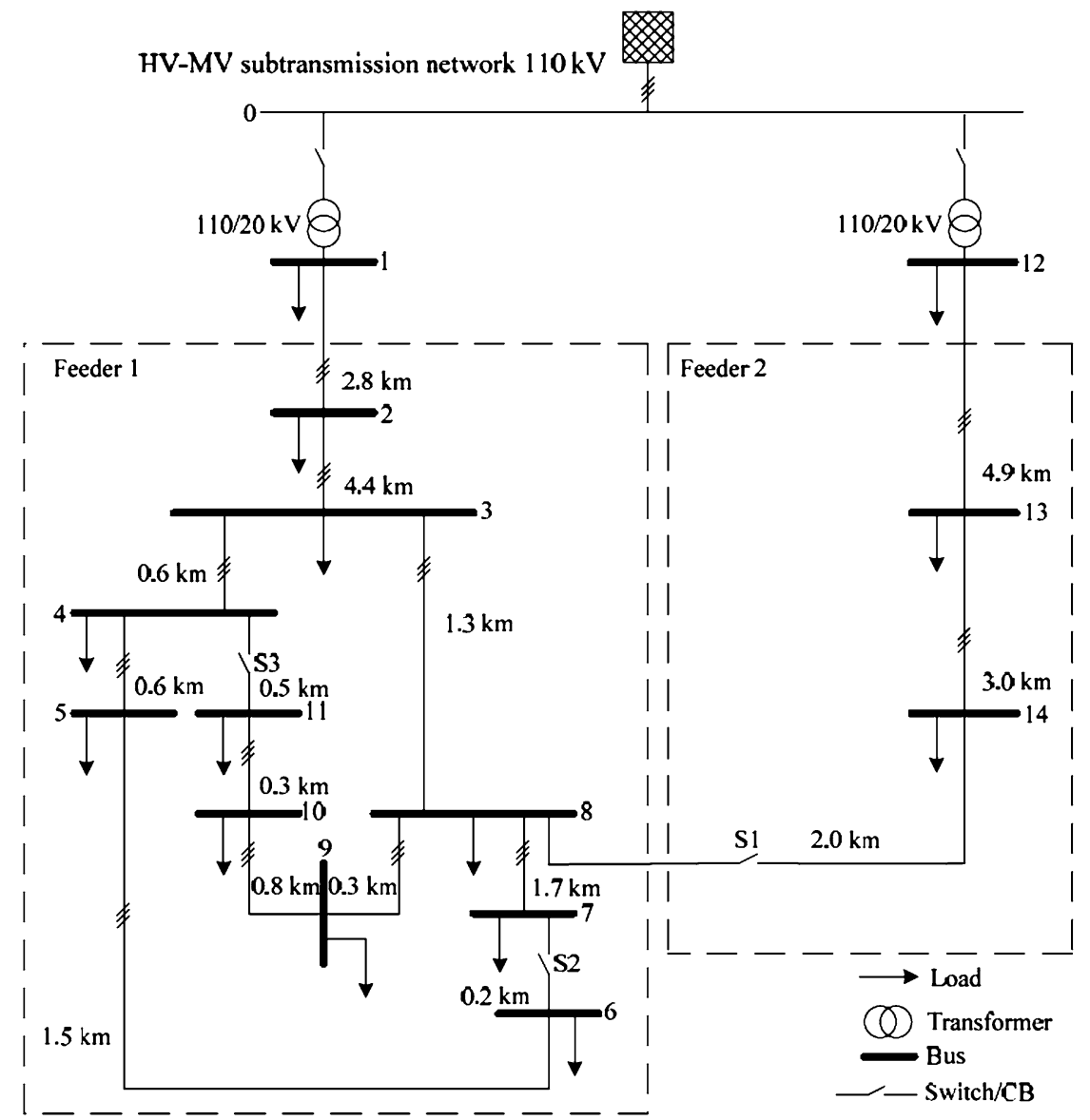

Abb. 8. Europäisches Mittelspannungsreferenznetz nach CIGRE [13]

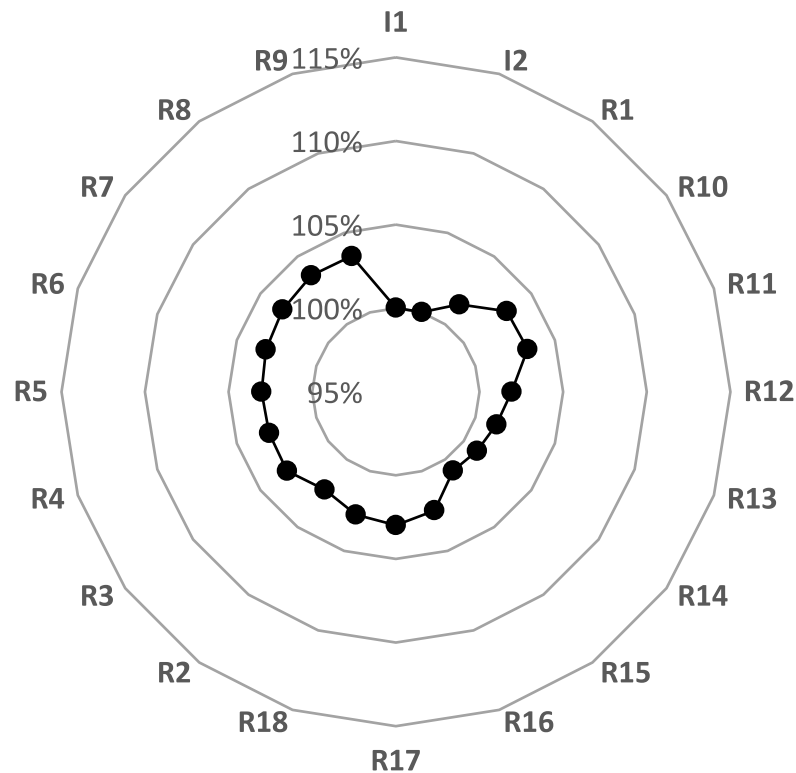

Abb. 9. Veränderung der dreipoligen Anfangs-Kurzschlusswechselströme aller Sammelschienen in Szenario 2 (Bezeichnung nach Abb. 7)

Aus den Ergebnissen geht hervor, dass die Größe der $l_{k}^{\prime \prime}$ max bei zunehmender Energieversorgung aus regenerativen Energiequellen

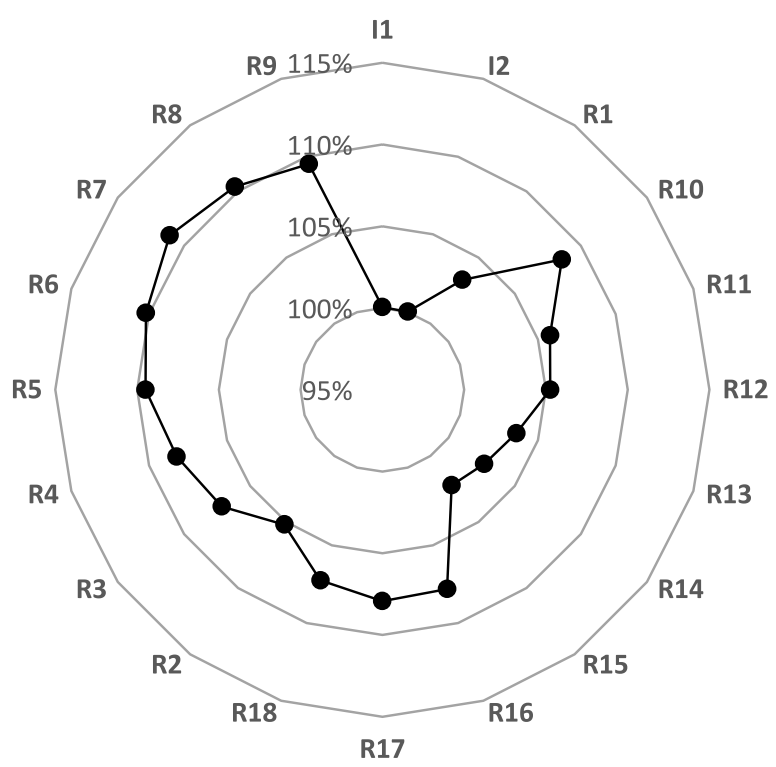

Abb. 10. Veränderung der dreipoligen Anfangs-Kurzschlusswechselströme aller Sammelschienen in Szenario 3

ansteigt. Der Durchschnittswert des Anstiegs an den Sammelschienen 11 bis R18 beträgt ca. 13,3\% im Szenario 4. Die Größe der Zunahme der $l_{k \text { max }}^{\prime \prime}$ variiert je nach Sammelschiene stark. In Berei- 


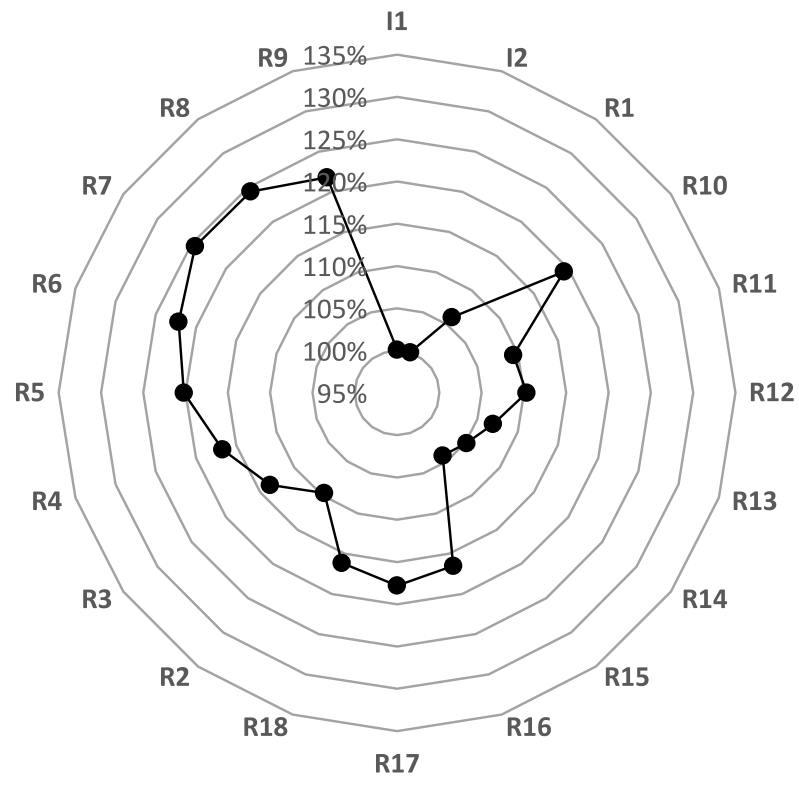

Abb. 11. Veränderung der dreipoligen Anfangs-Kurzschlusswechselströme aller Sammelschienen in Szenario 4

chen, in welchen keine regenerativen Erzeugungsanlagen integriert werden (z. B. I2) verändern sich die $l_{k \text { max }}^{\prime \prime}$ nur minimal (ca. 0,05\%). An den Sammelschienen R7 und R8 ist ein Anstieg um bis zu $25 \%$ erkennbar.

\subsection{Diskussion der Ergebnisse der NS}

Zusammengefasst zeigen die Simulationsergebnisse folgendes:

- Vergrößerung des Mittelwertes der $l_{k}^{\prime \prime}$ max bis zu $25 \%$ bei einem Ausbau der Lastversorgung durch EE - Anlagen bis zu $80 \%$

- Größerer Anstieg der $l_{k}^{\prime \prime}$ max bei größerer Distanz des Kurzschlusses zur Netzeinspeisung, weil die Leitungsimpedanz zunimmt und der Einfluss der Netzeinspeisung sinkt.

- Die zunehmende Integration von EE - Anlagen führt zu einer Vergrößerung der Kurzschlussströme in Niederspannungsverteilnetzen.

Der Zuwachs der $l_{k \max }^{\prime \prime}$ deutet auf eine zukünftige, notwendige Betrachtung der mechanischen Festigkeit der Sammelschienen hin. Die Ergebnisse der Simulation belegen die aufgestellte Hypothese, dass die zunehmende Integration von EE-Anlagen in der Energiewende zu einer positiven Veränderung, also einer Vergrößerung der Kurzschlussströme im Verteilnetz führt.

Der Vergleich mit den Ergebnissen der Studien ist nur bedingt möglich, da die Ergebnisgewinnung bei unterschiedlichen Ausgangsbedingungen erfolgt. Sie beziehen z. B. den Kurzschlussleistungsbetrag aus dem Ausland nicht mit ein. Der Anstieg der Kurzschlussleistung in der Verteilnetzebene aufgrund der Verlagerung der Erzeugung in diese Netzebene ist in der Simulation deutlich erkennbar. Die Ergebnisse ähneln dem, der dena Systemdienstleistungsstudie [8] in Bezug auf die Veränderung der Kurzschlussleistung eines ländlich geprägten Verteilnetzes. Die Ausgangspunkte beider Ergebnisse sind aufgrund der Vernachlässigung des Kurzschlussstrombeitrags aus dem Ausland und der Betrachtung eines kombinierten Netzes vergleichbar. Es werden große Schwankungen des Anstiegs der Kurschlussströme abhängig von der Sammelschiene deutlich und ein zukünftiger Anstieg der Kurzschlussströme erkennbar.

\section{Zusammenfassung}

Die Analysen zeigen, dass in den nächsten Jahren nicht damit zu rechnen ist, dass die Kurzschlussströme in der Niederspannung aufgrund der Abschaltung von Großkraftwerken, die an das Übertragungsnetz angeschlossen sind, sinken. Weiterhin verringern sich die Impedanzen der Netzkoppelungen der Spannungsebenen, da sich die Planungsgrundsätze durch den Zubau von EE-Anlagen, verändert haben; bei Ersatz von Transformatoren werden größere Bemessungsscheinleistungen angesetzt, da die Planung nicht mehr primär an der Gleichzeitigkeit der Lasten erfolgt.

Der Kurzschlussstrom kommt vermehrt aus der Fläche. Es ist davon auszugehen, dass der Kurzschlussstrom aufgrund des Zubaus von EE-Anlagen und der verbesserten Verkopplung der Spannungsebenen aufgrund von Netzverstärkungsmaßnahmen ansteigt. Erfahrungen der Netzbetreiber wie Stromnetz Hamburg, Mitnetz und Tennet zeigen, dass Betriebsmittel, wie regelbare Ortsnetztransformatoren kaum die Höhe des Kurzschlussstromes beeinflussen. Unabhängig davon wird dies durch weiterführende Untersuchungen bestätigt. Die wetterabhängigen bzw. tageszeitlichen Schwankungen der Kurschlussimpedanz des Übertragungsnetzes wirken sich nicht auf den Kurzschlussstrom der Verteilnetze in der Niederspannungsebene aus, dies zeigt die Berechnung aus Abschn. 2.2.

In weiteren Schritten sollten der Kurzschlussstrombeitrag aus dem Ausland und die lokalen Gegebenheiten eines beispielhaft gewählten Mittel- und Niederspannungsnetz untersucht werden. Dabei ist z. B. zu prüfen ob die vorhandene Fläche in dem gewählten Gebiet für den Zubau der EE-Anlagen geeignet ist. Insbesondere zwei Fälle könnten der zukünftigen Entwicklung der Kurzschlussströme interessant sein: „Blinding of Protection“ sowie ungewollte Inselnetzbildungen stellen die wohl größten Gefahren dar. Für die Bewertung dieser Szenarien müssen Parameterräume definiert und Multiparametermodelle verwendet werden.

Funding Note Open Access funding enabled and organized by Projekt DEAL.

Hinweis des Verlags Der Verlag bleibt in Hinblick auf geografische Zuordnungen und Gebietsbezeichnungen in veröffentlichten Karten und Institutsadressen neutral.

Open Access Dieser Artikel wird unter der Creative Commons Namensnennung 4.0 International Lizenz veröffentlicht, welche die Nutzung, Vervielfältigung, Bearbeitung, Verbreitung und Wiedergabe in jeglichem Medium und Format erlaubt, sofern Sie den/die ursprünglichen Autor(en) und die Quelle ordnungsgemäß nennen, einen Link zur Creative Commons Lizenz beifügen und angeben, ob Änderungen vorgenommen wurden. Die in diesem Artikel enthaltenen Bilder und sonstiges Drittmaterial unterliegen ebenfalls der genannten Creative Commons Lizenz, sofern sich aus der Abbildungslegende nichts anderes ergibt. Sofern das betreffende Material nicht unter der genannten Creative Commons Lizenz steht und die betreffende Handlung nicht nach gesetzlichen Vorschriften erlaubt ist, ist für die oben aufgeführten Weiterverwendungen des Materials die Einwilligung des jeweiligen Rechteinhabers einzuholen. Weitere Details zur Lizenz entnehmen Sie bitte der Lizenzinformation auf http://creativecommons.org/licenses/by/4.0/deed.de.

\section{Literatur}

1. Deutsche Bundesregierung (2018): Energiewende. Online. Available: https://www. bundesregierung.de/Webs/Breg/DE/Themen/Energiewende/Fragen-Antworten/1_ Allgemeines/1_warum/_node.html. (Zugriff am 12 März 2018).

2. Deutsches Atomforum (2018): www.kernenergie.de. Online. Available: http:// www.kernenergie.de/kernenergie/themen/strom/Zahlen-und-Fakten/01_index.php\# anchor_f93853ce_Accordion-Kernkraftwerke-in-Betrieb. (Zugriff am 12 März 2018).

3. VDE-AR-N 4105 Anwendungsregel (2018-11): Erzeugungsanlagen am Niederspannungsnetz - Technische Mindestanforderungen für Anschluss und Parallelbetrieb von Erzeugungsanlagen am Niederspannungsnetz. 
4. Grumm, F., Plenz, M., Meyer, M. F., Jordan, M., Kaatz, G., Schulz, D. (2018): Influence of PV-systems on short-circuit currents in low-voltage distribution grids in structurally weak areas. In 2018 IEEE international conference on environment and electrical engineering and 2018 IEEE industrial and commercial power systems Europe (EEEIC I\&CPS Europe), Palermo, Italy, 12-15 June 2018. https://doi.org/10.1109/EEEIC.2018. 8493921.

5. Grumm, F., Plenz, M., Meyer, M. F., Schulz, D. (2019): Investigation of technologies and parameters influencing the short-circuit current level of low-voltage distribution networks. In NEIS 2018, conference on sustainable energy supply and energy storage systems, Hamburg, Germany, 20-21 September 2018 (S. 285-292). Berlin: IEEE Conference VDE Verlag. ISBN 978-3-8007-4821-1.

6. Valov, B. (2013): Änderung der Kurzschlussleistungen. Künftiges Energieversorgungs system gemäß NEP 2012. Magazin Energie-Wirtsch., 4(2013), 50-55.

7. Janssen, A. L., van Riet, M., Smeets, R. P., Kanters, J., van den Akker, W. F., Aanhaanen, G. L. (2012): Prospective single and multi-phase short-circuit current levels in the Dutch transmission, sub-transmission and distribution grids. In CIGRE conference, Paris.

8. Deutsche Energie-Agentur GmbH (dena) (2014): dena-Studie Systemdienstleistung 2030: Sicherheit und Zuverlässigkeit einer Stromversorgung mit hohem Anteil. Berlin: Deutsche Energie-Agentur GmbH (dena).

9. Kühn, H. (2018): Sicherstellung der Schutzfunktion in AC-Netzen. ETG J., 1(2018), 15-18.

10. FGH (2012): Studie zur Ermittlung der technischen Mindesterzeugung des konventionellen Kraftwerksparks zur Gewährleistung der Systemstabilität in den deutschen

\section{Autoren}

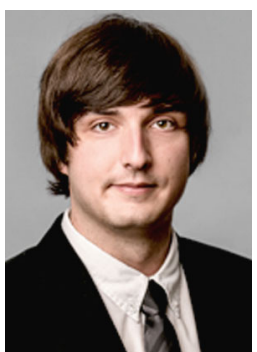

\section{Maik Plenz}

Studium des Wirtschaftsingenieurwesens mit der Fachrichtung Energielogistik an der Hochschule Lausitz / BTU Cottbus-Senftenberg, Abschluss 2012. Seit 2013 an der BTU Cottbus-Senftenberg, Leuphana Universität Lüneburg und aktuell an der HSU Hamburg am Fachgebiet Elektrische Energiesysteme. Von 2017 bis 2019 war er Akademischer Mitarbeiter bei Hamburg Innovation $\mathrm{GmbH}$ und beendete 2019 einen Forschungsaufenthalt an der University of Oxford.

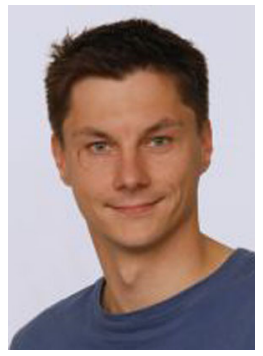

\section{Florian Grumm}

absolvierte sein Diplomstudium in der Fachrichtung Elektrotechnik mit dem Schwerpunkt Steuerungs- und Regelungstechnik an der Technischen Universität Berlin. Zurzeit arbeitet er am Fachbereich Elektrische Energiesysteme an der Helmut-Schmidt-Universität / Universität der Bundeswehr Hamburg und forscht in den Bereichen Flugzeugnordnetzen und Netzrückwirkungen.

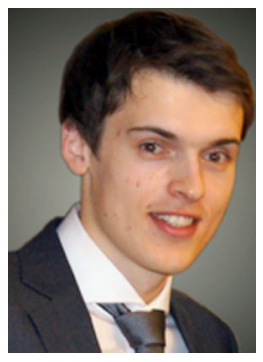

\section{Marc Florian Meye}

absolvierte sein Masterstudium in der Fachrichtung Elektrotechnik an der Technischen Universität Hamburg im Jahr 2016. Zurzeit arbeitet er am Fachbereich Elektrische Energiesysteme an der Helmut-Schmidt-Universität / Universität der Bundeswehr Hamburg und forscht in den Bereichen Netzintegration von Erneuerbaren Energieanlagen und Netzrückwirkungen.
Übertragungsnetzen bei hoher Einspeisung aus erneuerbaren Energien. Mannheim: Forschungsgemeinschaft für Elektrische Anlagen und Stromwirtschaft (FGH) e. V.

11. Universität Kassel Studie (2014): Studie zur Veränderung der Kurzschlussleistung beim Übergang von einem zentral zu einem dezentral gespeisten Energieversorgungssystem: Endbericht zum Verbundvorhaben. Kassel.

12. Heuck, K., Dettmann, K.-D., Schulz, D. (2013): Erzeugung, Übertragung und Verteilung elektrischer Energie für Studium und Praxis. Wiesbaden: Vieweg+Teubner Verlag | Springer Fachmedien Wiesbaden GmbH.

13. CIGRE task force (2014): Benchmark Systems for Network Integration of Renewable and Distributed Energy Resources.

14. IEC 609090-0:2016: Kurzschlussströme in Drehstromnetzen - Teil 0: Berechnung der Ströme.

15. Schürhuber, R. (2016): Die Kurzschlussnorm IEC 60909-0: 2016 - Neues und Änderungen. E\&l, Elektrotech. Inf.tech., 133(4-5), 228-235. https://doi.org/10.1007/ s00502-016-0416-8.

16. Fuchs, B. et al.: Studie zu Aspekten der elektrischen Systemstabilität im deutschen Übertragungsnetz bis 2023: Abschlussbericht; [Gutachten des Institut für Hochspannungstechnik der RWTH Aachen University]; eine Studie im Auftrag der Bundesnetzagentur ... zur Ausschreibung: „Ermittlung optimaler Standorte für netzstützende Erzeugungseinheiten", BNA-2015, Aachen. Online: https://publications.rwth-aachen. de/record/538613.

17. Pistora, G. (2016): Berechnung von Kurzschlussströmen und Spannungsfällen. Berlin: VDE Verlag GmbH. ISBN 978-3-8007-4224-0.

\section{Scott Boden}

Studium des Wirtschaftsingenieurwesens mit der Fachrichtung Energielogistik an der Hochschule Lausitz / BTU Cottbus-Senftenberg, Abschluss 2018.

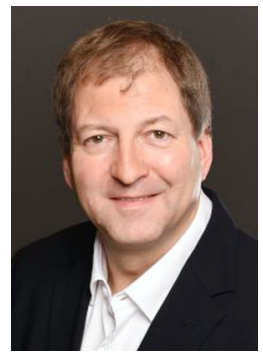

Detlef Schulz

Univ.-Prof. Dr.-Ing. habil. Detlef Schulz, Dipl.Ing., leitet das Fachgebiet Elektrische Energiesysteme an der Helmut-Schmidt-Universität/Universität der Bundeswehr Hamburg. Er promovierte und habilitierte an der TU Berlin in den Jahren 2002 und 2006. Seine Forschungsgebiete umfassen die Netzintegration erneuerbarer Energien, Netzberechnung und Netzausbau, Energiespeicher sowie Flugzeugbordnetze.

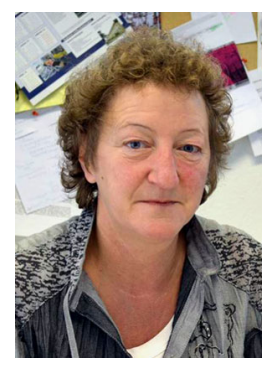

\section{Kathrin Lehmann}

Prof. Dr.-Ing. Kathrin Lehmann leitet das Fachgebiet Elektrische Energietechnik und ist Prodekanin der Fakultät 3 - Maschinenbau, Elektro- und Energiesysteme an der BTU Cottbus-Senftenberg. Sie promovierte 1988 im Fachgebiet der Elektroenergietechnik an der TU Dresden. Ihre Forschungsschwerpunkte umfassen die Energielogistik, Systemdienstleistungen und Netzplanung. 